\title{
Uso popular de plantas medicinais na cicatrização de feridas: implicações para a enfermagem
}

\section{Traditional use of medicinal plants to heal wounds: implications for nursing}

\section{Uso popular de plantas medicinales en la cicatrización de heridas: implicaciones para el equipo} de enfermería

\author{
Manuelle Arias Piriz'; Adriana Roese ${ }^{\text {II }}$; Caroline Vasconcellos Lopes ${ }^{I I I}$; Marcelo Melo Silva ${ }^{I V}$; \\ Rita Maria HeckV; Rosa Lia Barbieri ${ }^{\mathrm{VI}}$
}

\begin{abstract}
RESUMO: Este estudo teve como objetivo identificar as plantas medicinais indicadas por informantes folk do sul do Brasil para o tratamento de feridas, explicitando as implicações para a prática profissional da enfermagem. Trata-se de estudo qualiquantitativo, descritivo, realizado em quatro municípios do sul do Brasil. Foi realizada entrevista semiestruturada gravada, com georreferenciamento dos locais de coleta, observação sistemática e registro fotográfico das plantas medicinais. Foram entrevistados 10 informantes, entre setembro de 2009 e agosto de 2011. Os resultados evidenciaram a indicação de 19 plantas medicinais para o tratamento de feridas. Destas, 16 possuem efeitos cicatrizantes relatados na literatura científica. Assim, a enfermagem, profissão que valoriza o cuidado às feridas, necessita entender como este cuidado é prestado popularmente, para que o enfermeiro torne-se sujeito ativo na recuperação da saúde da população atendida.
\end{abstract}

Palavras-Chave: Fitoterapia; cuidados de enfermagem; medicina tradicional; cicatrização.

\begin{abstract}
A qualitative and quantitative, descriptive study was conducted in four cities in southern Brazil to identify the medicinal plants indicated by folk informants for treating wounds, and the implications for professional nursing practice. Between September, 2009 and August, 2011, semi-structured interviews of ten informants were recorded, medicinal plant collection sites were georeferenced, with systematic observation, and photographic records were made. Results showed that 19 medicinal plants were being indicated for treating wounds. Of these, the healing effects of 16 are reported in the scientific literature. Accordingly, nursing, a profession that appreciates the importance of caring for wounds, should understand how this care is provided traditionally, in order for nurses to become active subjects in restoring health in the population served.

Keywords: Phytotherapy; nursing care; traditional medicine; healing.

RESUMEN: Este estudio tuvo como objetivo identificar las plantas medicinales indicadas por informantes folk del sur de Brasil para el tratamiento de heridas, explicitando las implicaciones para la práctica profesional de la enfermería. Se trata de un estudio cualitativo y cuantitativo, descriptivo, realizado en cuatro ciudades del sur de Brasil. Se llevaron a cabo la entrevista semiestructurada grabada, la georreferenciación de los sitios de recolección, la observación sistemática y el registro fotográfico de las plantas medicinales. Se entrevistaron diez informantes entre septiembre de 2009 y agosto de 2011. Los resultados mostraron que 19 plantas medicinales están indicadas para el tratamiento de heridas. Entre estas, literatura científica ha relatado que 16 tienen efectos de cicatrización. Por lo tanto, la enfermería, profesión que valora el cuidado de las heridas, necesita comprender cómo se hace este cuidado popularmente, para que el enfermero se vuelva un sujeto activo en la recuperación de la salud de la población atendida. Palabras Clave: Fitoterapia; cuidados de enfermería; medicina tradicional; cicatrización.
\end{abstract}

\section{INTRODUÇÃO}

O emprego de plantas medicinais no processo de cicatrização de feridas é mencionado desde a pré-história, em que eram utilizados plantas e extratos vegetais para o cuidado humano. $\mathrm{O}$ intuito era estancar hemorragias e favorecer a cicatrização, sendo que muitas dessas plantas eram ingeridas para atuação em via sistêmica ${ }^{1}$.

Ainda hoje, é de grande importância reconhecer as formas de cuidado realizadas pelas pessoas em seu cotidiano, revelando-se a necessidade de um maior

'Enfermeira. Mestre em Enfermagem. Doutoranda pelo Programa de Pós-Graduação em Enfermagem da Universidade Federal de Pelotas. Rio Grande do Sul, Brasil.E-mail: manuelle.piriz@hotmail.com.

"Enfermeira. Doutora em Enfermagem. Professora Adjunta da Faculdade de Enfermagem da Universidade Federal do Rio Grande do Sul. Porto Alegre, Rio Grande do Sul, Brasil. E-mail: adiroese@gmail.com.

IIIEnfermeira. Mestre em Enfermagem. Doutoranda do Programa de Pós-Graduação em Enfermagem da Universidade Federal de Pelotas. Rio Grande do Sul, Brasil. E-mail: carolinevaslopes@gmail.com.

IVEnfermeiro. Graduado pela Universidade Federal de Pelotas. Rio Grande do Sul, Brasil. E-mail: marcello_melo@yahoo.com.br.

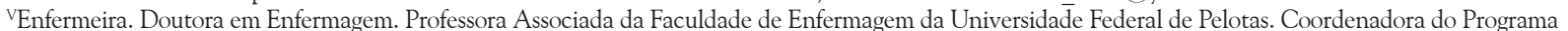
de Pós-Graduação em Enfermagem. Pelotas, Rio Grande do Sul, Brasil. E-mail: rmheckpillon@yahoo.com.br.

v'Bióloga. Doutora em Genética e Biologia Molecular. Pesquisadora da Empresa Brasileira de Pesquisa Agropecuária. Pelotas, Rio Grande do Sul, Brasil. E-mail: barbieri.cpact@emprapa.com.br. 
domínio desse saber pelos profissionais de enfermagem. Isso porque a comunidade é um espaço do conhecimento popular que pode ser utilizada como um instrumento de proximidade, autonomia e de valorização da cultura de cada ser humano cuidado ${ }^{2}$.

profissional enfermeiro, principalmente, deve atentar tanto para essas questões culturais da comunidade quanto para a multidimensionalidade do ser humano ${ }^{3}$. Desse modo, é necessário levar em consideração o compromisso com o cuidar, o que pode favorecer não apenas diagnósticos, resultados e intervenções de enfermagem, mas todo o planejamento da prática, tornando o convívio das pessoas com a ferida menos dolorido.

Desta forma, por meio da aproximação e compreensão do universo de cuidado cotidiano de vida do agricultor e informante folk (pessoas que detêm um grande conhecimento sobre as plantas e que também podem ser chamados de curandeiros, raizeiros ou erveiros), será possível estabelecer uma terapêutica eficaz que auxilie no cuidado prestado. $\bigcirc$ saber popular deve ser compreendido e acrescido de conhecimentos e atitudes respaldados pelo saber científico, ampliando as pesquisas sobre a utilização de plantas medicinais na perspectiva de garantir uma assistência integral e acolhedora por parte dos profissionais do Sistema Único de Saúde (SUS) ${ }^{4}$.

O importante é que o conhecimento sobre plantas medicinais possa contribuir, efetivamente, na área da saúde, suprindo as necessidades básicas de cada população, em especial no que tange ao tratamento de feridas, prática tão familiar à enfermagem ${ }^{5}$.

Objetivo deste estudo foi identificar as plantas medicinais indicadas por informantes folk do sul do Brasil para o tratamento de feridas, explicitando as implicações para a prática profissional da enfermagem.

\section{REVISÃO DE LITERATURA}

Para a antropologia interpretativa, a cultura é construída pelos sujeitos ao interagirem no cotidiano, surgindo, dessa inter-relação, valores, formas de cuidado, trocas de saberes sobre as plantas medicinais, crenças e normas que fazem parte da práxis. Assim, a cultura pode ser conceituada como um sistema de símbolos compartilhados, que as pessoas usam para perceber, interpretar e organizar o mundo em que vivem ${ }^{6}$.

Nessa perspectiva, pode-se entender a especificidade de determinado grupo com relação à identificação de uma planta que, pela experiência, é empregada para aliviar um determinado sintoma. Em outro grupo, pode ser que esta mesma planta seja utilizada no cuidado à saúde para outro sintoma. Assim, a planta medicinal é um símbolo compartilhado, que só tem sentido a partir do seu reconhecimento visual, olfativo e curativo acumulado e repassado entre as gerações.
Desse modo, entende-se que, para discutir o cuidado, é fundamental aproximá-lo da cultura, enfatizando que os sistemas de cuidado à saúde são cultural e socialmente construídos. Estes sistemas de cuidado são formas de reproduzir a realidade social que imprime significado às interações existentes entre pessoas ${ }^{7,8}$.

Existe, portanto, um modelo explicativo de cuidado à saúde que atenta para o fato de que dentro de uma mesma sociedade coexistem diferentes sistemas de saúde, com uma multiplicidade de concepções sobre a doença, incluindo etiologia, fisiopatologia, definição de severidades, tratamento e diagnóstico. Nesse contexto, diferenciam-se três sistemas básicos de cuidado à saúde?

- Sistema de cuidado profissional (oficial) - que corresponde ao sistema médico instituído e aceito como formal nas sociedades ocidentais - o modelo biomédico. Este sistema compreende as práticas e os saberes que são constituídos por um conhecimento científico ou por uma tradição específica de cuidados à saúde, como a biomedicina, a homeopatia e a medicina chinesa.

- Sistema de cuidado popular (familiar) - composto por conhecimento leigo, referente aos saberes e práticas cotidianas que estão relacionadas ao fenômeno saúde-doença. É neste contexto que os primeiros cuidados com a saúde e a doença são adotados.

- Sistema de cuidado folk (popular) - refere-se aos especialistas de cura não reconhecidos legalmente, que utilizam recursos como plantas medicinais, tratamentos manipulativos, exercícios especiais e considerados como sagrados quando envolvem o xamanismo e os rituais de cura. Estes especialistas são legitimados pela sociedade e estão fortemente ligados ao sistema de cuidado familiar. A maioria dos cuidadores folk compartilha os mesmos valores culturais e a mesma visão de mundo das comunidades onde vivem, oferecendo explicações sobre a enfermidade mais acessíveis à compreensão do indivíduo e sua família ${ }^{7,8}$.

Dessa forma, este estudo busca aproximar-se do sistema de cuidado folk, a fim de valorizar a cultura popular e conhecer as práticas de cuidado às feridas utilizadas por representantes/especialistas do sistema informal de saúde, aproximando estes saberes da prática profissional da enfermagem contemporânea.

\section{Metodologia}

Foi realizado um estudo qualiquantitativo e descritivo 9 , o qual faz parte do projeto de pesquisa Plantas bioativas de uso humano por famílias de agricultores de base ecológica da Região Sul do Rio Grande do Sul, enfocando o uso de plantas medicinais utilizadas na cicatrização de feridas indicadas por pessoas que são referência no conhecimento de plantas medicinais, em comunidades rurais. 
Foram entrevistadas 10 pessoas, indicadas por agricultores ecológicos e pela Associação Riograndense de Empreendimentos de Assistência Técnica e Extensão Rural (Emater) ou mesmo pelas equipes da Estratégia de Saúde da Família das localidades. Destas, sete foram abordadas no domicílio e as demais, no local onde prestavam atendimento ao público, nos Municípios de Pelotas, Canguçu, Morro Redondo e São Lourenço do Sul, que se localizam na Região Sul do Rio Grande do Sul.

Durante a primeira visita aos informantes, foi feito o convite formal para participação na pesquisa e estabelecida uma agenda de encontros, que variou de dois a doze. Os instrumentos de pesquisa para coleta de dados foram entrevista semiestruturada gravada e observação sistemática9 .

Somente um informante folk não concordou com o uso do gravador. Neste caso, a abordagem foi realizada com pelo menos duas pessoas para auxiliar nas anotações dos registros.

Foi realizado registro fotográfico in loco das plantas medicinais citadas para posterior identificação taxonômica. Quando houve dúvida na correta identificação de algumas plantas, com a autorização do informante folk, foram coletados ramos em fase reprodutiva para preparação de exsicatas. Os locais das entrevistas e as plantas citadas foram georreferenciados por meio de Global Positioning System (GPS) de navegação. A coleta de dados ocorreu entre setembro de 2009 e agosto de 2011. Para este artigo, foram selecionadas as plantas indicadas como medicinais para o tratamento de feridas por, no mínimo, três informantes. E para a análise dos dados, realizou-se revisão de literatura para cada planta medicinal utilizada, buscando confrontar suas indicações com os estudos científicos existentes.

Foram respeitados todos os preceitos éticos e legais da pesquisa em enfermagem, bem como a Resolução $n^{\circ} 466 / 12$ de competência do Conselho Nacional de Saúde, que emana diretrizes sobre a pesquisa com seres humanos $^{10}$.

Os participantes da pesquisa assinaram o Termo de Consentimento Livre e Esclarecido em duas vias. O projeto recebeu parecer favorável de um Comitê de Ética e Pesquisa da área de saúde.

\section{Resultados e Discussão}

Segundo os 10 participantes da pesquisa, 19 plantas medicinais podem ser utilizadas no tratamento de feridas por possuírem efeitos cicatrizantes. Com tais informações, os autores deste estudo elaboraram a Figura 1, complementando as especificações necessárias, com apoio de literatura.

Na literatura científica, foram encontrados relatos da atividade cicatrizante específica para 16 plantas das
19 espécies medicinais citadas pelos informantes folk, ou seja, a maioria delas.

O Allium satiuum (alho) tem comprovado efeito na cicatrização de feridas ${ }^{11}$. Várias espécies do gênero Aloe (babosa) também possuem efeitos cicatrizantes ${ }^{12}$, com maior destaque para Aloe vera ${ }^{13}$. Foram demonstradas atividades antibacterianas em endodontia para a espécie Arctium lappa (bardana) ${ }^{14,15}$. A aplicação tópica de gel elaborado a partir do óleo de Calendula officinalis (calêndula) demonstrou capacidade de reepitelização e cicatrização de feridas ${ }^{16}$. Por sua vez, a pomada elaborada com o extrato de Melia azedarach (cinamomo) mostrou ação antibacteriana e anti-inflamatória em feridas incisionais, as quais cicatrizaram em torno de 15 dias $^{17}$.

A ação local de Symphytum officinale (confrei) está relacionada à presença da lantoína - substância de comprovada ação cicatrizante. Em estudo realizado com 161 pacientes com úlceras de pressão, a pomada creme com extrato de confrei obteve um percentual de $90,4 \%$ de cicatrização em um período de quatro semanas ${ }^{18}$.

Foram encontradas evidências da ação cicatrizante para Polygonum barbatum e Polygonum cuspidatum (erva-de-bicho $)^{19,20}$. Em relação à eficácia e à segurança do uso de Solanum americanum (erva-moura), ainda não houve testes em estudos científicos e sua utilização tem sido feita com base no conhecimento popular ${ }^{21}$.

Por sua vez, Maytenus ilicifolia (espinheira-santa) tem efeito protetor contra lesões gástricas, por meio da inibição de secreção do suco gástrico ${ }^{22}$. Além disso, atua na cicatrização de lesões estomacais e é fonte de agentes antioxidantes ${ }^{23}$.

Para Bryophyllum pinnatum (folha-da-fortuna) foi realizado um estudo experimental em ratos, sendo comprovada sua eficácia anti-inflamatória e antinociceptiva $^{24}$. Estudo experimental em ratos revelou que o extrato hidroalcoólico de Stachytarpheta cayennensis (gervão) apresenta propriedades anti-inflamatórias e antinociceptivas em uso externo ou interno ${ }^{25}$. Além disso, foi comprovado seu potencial antibacteriano ${ }^{26}$.

Para Citrus sinensis (laranjeira), o extrato da casca da planta, administrado de forma oral, apresentou efeitos significativos na cicatrização de feridas em ratos com diabetes induzida, em decorrência da grande concentração de vitamina $\mathrm{C}$ e carotenoides ${ }^{27}$.

A planta Malva sylvestris (malva) apresentou resultados eficazes na cicatrização de feridas ${ }^{28}$. Estudos farmacológicos com a espécie Achyrocline satureioides (marcela) comprovaram atividade anti-inflamatória, analgésica, imunoestimulante, antiviral, antimicrobiana, hipoglicemiante e antioxidante ${ }^{29,30}$.

Não foram encontrados estudos específicos de utilização da espécie Senecio brasiliensis (maria-mole) na saúde humana. Entretanto, um estudo realizado na África com outra espécie do mesmo gênero, Senecio serratuloides, concluiu que o extrato da planta 
apresenta boa capacidade de regeneração tecidual em feridas profundas ${ }^{31}$.

Foi demonstrado potencial antibacteriano, anti-inflamatório, antioxidante e analgésico para Piper umbellatum (pariparoba) ${ }^{32}$. Além disso, esta espécie apresenta alta concentração de polifenóis e atividade antioxidante, com ação contra os radicais livres ${ }^{33}$.

A espécie Alternanthera dentata (penicilina) apresentou significativa citotoxicidade em tumores e consideráveis efeitos antitumorais ${ }^{17}$, porém não foram encontrados estudos que comprovassem seus efeitos cicatrizantes.
O óleo essencial de Schinus molle (periquiteira) demonstrou propriedades antimicrobianas, antifúngicas, anti-inflamatórias e cicatrizantes ${ }^{34}$.

A análise farmacológica dos extratos hidroalcóolicos de folhas, raízes e sementes de Plantago australis (tansagem) comprovou sua ação analgésica e anti-inflamatória ${ }^{35}$. Outra espécie próxima, Plantago ovata, tem ação antisséptica na limpeza de feridas, anti-inflamatória e antialérgica ${ }^{36}$. Polifenóis extraídos de folhas e sementes de Plantago major, outra espécie do mesmo gênero, demonstraram efeitos na cicatrização de feridas; além disso, apresentam capacidade

\begin{tabular}{|c|c|c|c|c|}
\hline $\begin{array}{l}\text { Nome } \\
\text { popular }\end{array}$ & $\begin{array}{l}\text { Nome } \\
\text { científico }\end{array}$ & $\begin{array}{c}\text { Parte } \\
\text { utilizada }\end{array}$ & Forma de uso & Dose \\
\hline Alho & $\begin{array}{l}\text { Allium } \\
\text { sativum }\end{array}$ & Bulbilho & $\begin{array}{l}\text { Tintura; tomar gotas. Em forma } \\
\text { de maceração de meio bulbo de } \\
\text { alho enrolado na gaze, colocar } \\
\text { na lesão ou utilizar na comida. }\end{array}$ & $\begin{array}{l}\text { Colocar em cima da lesão o } \\
\text { alho por } 3 \text { dias. Ou } 80 \text { gotas de } \\
\text { tintura de } 2-3 \text { vezes ao dia. }\end{array}$ \\
\hline Babosa & Aloe spp. & Folha & $\begin{array}{l}\text { Utilizar a folha sem casca direta- } \\
\text { mente sobre a queimadura ou } \\
\text { ferimento. }\end{array}$ & $2-3$ vezes ao dia. \\
\hline Bardana & Arctium lappa & Folha & $\begin{array}{l}\text { Sabonete com a tintura da planta } \\
\text { para lavar ferida. }\end{array}$ & 2 vezes ao dia. \\
\hline Calêndula & $\begin{array}{l}\text { Calendula } \\
\text { officinalis }\end{array}$ & $\begin{array}{l}\text { Flor, talo, } \\
\text { folha. }\end{array}$ & $\begin{array}{l}\text { Pomada, infusão ou óleo para } \\
\text { passar na pele. }\end{array}$ & $\begin{array}{l}\text { Lavar } 2 \text { vezes ao dia. Por três } \\
\text { dias. }\end{array}$ \\
\hline Cinamomo & $\begin{array}{c}\text { Melia } \\
\text { azedarach }\end{array}$ & $\begin{array}{l}\text { Casca do } \\
\text { Tronco }\end{array}$ & Decocção para lavar a ferida. & Lavar 3 vezes ao dia. \\
\hline Confrei & $\begin{array}{l}\text { Symphytum } \\
\text { officinale }\end{array}$ & Folha & Infusão para lavar a ferida. & n.i. \\
\hline Erva-de-bicho & Polygonum sp. & $\begin{array}{l}\text { Toda a } \\
\text { planta }\end{array}$ & Pomada. & n.i. \\
\hline Erva-moura & $\begin{array}{c}\text { Solanum } \\
\text { americanum }\end{array}$ & $\begin{array}{l}\text { Toda a } \\
\text { planta }\end{array}$ & $\begin{array}{l}\text { Maceração com um pouco de sal } \\
\text { iodado e com óleo de oliva ou } \\
\text { de girassol. }\end{array}$ & n.i. \\
\hline $\begin{array}{l}\text { Espinheira- } \\
\text { santa }\end{array}$ & $\begin{array}{l}\text { Maytenus } \\
\text { ilicifolia }\end{array}$ & Folha & $\begin{array}{l}\text { Tintura, infusão ou decocção } \\
\text { para uso interno. }\end{array}$ & $\begin{array}{l}\text { Usar } 3 \text { vezes ao dia, antes das } \\
\text { refeições, até melhorar. Utilizar } \\
\text { no máximo de } 15-20 \text { dias e dá } \\
\text { um intervalo de um mês. }\end{array}$ \\
\hline $\begin{array}{l}\text { Folha-da- } \\
\text { fortuna }\end{array}$ & $\begin{array}{l}\text { Bryophyllum } \\
\text { sp. }\end{array}$ & Folha & $\begin{array}{l}\text { Lavar a folha e aplicar no local } \\
\text { afetado. }\end{array}$ & Três vezes ao dia. \\
\hline Gervão & $\begin{array}{l}\text { Stachytarpheta } \\
\text { cayennensis }\end{array}$ & Folha & Infusão para uso interno. & Tomar durante o dia. \\
\hline Laranjeira & Citrus sinensis & Folha & Cataplasma. & $\begin{array}{l}\text { Utilizar umas } 3 \text { folhas e retirar } \\
\text { as nervuras das folhas. Aplicar } \\
\text { na ferida } 2 \text { vezes ao dia. }\end{array}$ \\
\hline Malva & $\begin{array}{c}\text { Malva } \\
\text { sy/vestris }\end{array}$ & Folha & Infusão para lavar a ferida. & 1 colher para uma xícara. \\
\hline Marcela & $\begin{array}{l}\text { Achyrocline } \\
\text { satureioides }\end{array}$ & $\begin{array}{l}\text { Flor ou } \\
\text { folha }\end{array}$ & Infusão para uso externo. & $\begin{array}{l}3 \text { a } 4 \text { vezes ao dia no período } \\
\text { máximo de uma semana. }\end{array}$ \\
\hline Maria-mole & $\begin{array}{c}\text { Senecio } \\
\text { brasiliensis }\end{array}$ & Flor & Infusão para uso externo. & n.i. \\
\hline Pariparoba & Piper sp. & Folha & Infusão para lavar a ferida. & 1 folha inteira ao dia. \\
\hline Penicilina & $\begin{array}{l}\text { Alternanthera } \\
\text { dentata }\end{array}$ & Folha & Infusão. & n.i. \\
\hline $\begin{array}{l}\text { Periquiteira } \\
\text { Tansagem }\end{array}$ & $\begin{array}{l}\text { Schinus molle } \\
\text { Plano sp. }\end{array}$ & $\begin{array}{l}\text { Flor } \\
\text { Folha e } \\
\text { semente }\end{array}$ & $\begin{array}{l}\text { Infusão para uso externo. } \\
\text { Infusão ou decocção para uso } \\
\text { externo e/ou interno. }\end{array}$ & $\begin{array}{l}\text { n.i. } \\
\text { Ingerir de } 3 \text { a } 4 \text { xícaras ao dia. }\end{array}$ \\
\hline
\end{tabular}

n.i. = não informado

FIGURA 1: Plantas medicinais indicadas para o tratamento de feridas. Pelotas, Rio Grande do Sul, 2015. 
antiúlcera, anti-inflamatória, antioxidante, atividade anticarcinogênica e antiviral ${ }^{37,38}$.

A Agência Nacional de Vigilância Sanitária (ANVISA) regulamenta, por meio da Resolução da Diretoria Colegiada (RDC) número 10, o uso de 66 plantas no cuidado da saúde humana. Entre estas, sete (Polygonum punctatum, Maytenus ilicifolia, Arctium lappa, Malva sylvestris, Calendula officinalis, Achyrocline satureioides e Plantago major), das 19 plantas citadas pelos informantes, são indicadas por possuírem efeito cicatrizante ${ }^{39}$.

Nesta perspectiva, os resultados mostram que, das 19 plantas analisadas como cicatrizantes, 16 (84,21\%) possuem estudos experimentais ou farmacológicos, que demonstram seu efeito cicatrizante. Dentre estas, seis apresentaram efeito diretamente na cicatrização de feridas e 10 atuam na cicatrização por meio de seu potencial anti-inflamatório ou antimicrobiano, considerando que as feridas infectadas ou inflamadas levam mais tempo para a sua cicatrização.

Com isso, pode-se verificar que a maioria das plantas indicadas pelos informantes folk para tratar feridas tem efeitos cicatrizantes relatados na literatura científica. Entretanto, para três plantas, não foram encontrados estudos relatando ação cicatrizante, o que, de modo algum, significa que elas não possuam esse efeito. Pode ocorrer que ainda não tenham sido realizados estudos científicos investigando esta ação.

No que tange à enfermagem, entre suas ações, destacam-se os cuidados com as feridas e o controle do processo de cicatrização. Os profissionais de enfermagem, tanto em serviços de atenção primária, secundária como terciária, devem atentar para a investigação dos fatores locais, sistêmicos e externos que contribuem para o surgimento e a cronicidade das lesões, interferindo no processo de cicatrização e visando a uma abordagem integral ${ }^{40}$.

Para que ocorra esta abordagem integral e contextualizada, é necessário que a enfermagem vincule o seu conhecimento com os saberes populares acerca das plantas medicinais utilizadas como cicatrizantes. Desse modo, surge uma nova perspectiva de cuidado, que valoriza os sistemas culturais e desenvolve novos saberes e habilidades para a melhoria dessa prática em saúde.

\section{Conclusão}

presente estudo possibilitou conhecer as práticas para o cuidado às feridas utilizadas pelos profissionais do sistema informal de saúde na Região Sul do Rio Grande do Sul. Entre os principais achados, destaca-se que a maioria das plantas citadas possui comprovação científica de sua eficácia na cicatrização. Entre as limitações do estudo, salienta-se a importância da continuidade do trabalho junto às comunidades, o que ainda não é realizado de forma efetiva, bem como a articulação entre usuários de plantas medicinais e universidade - esta, na forma de retorno das publicações científicas sobre o tema, para socializar conhecimentos e experiências.

Os informantes folk do Sul do Brasil pesquisados indicam corretamente, em sua maioria, as plantas medicinais, o que tenciona nosso olhar para a importância do resgate desses saberes por parte da enfermagem, profissão que valoriza o cuidado às feridas.

Assim, aliando-se ao cuidado popular e às pessoas que são referência neste assunto, o enfermeiro amplia suas competências de cuidar e pode tornar-se sujeito ativo na recuperação e promoção da saúde dos indivíduos.

\section{REFERÊNCIAS}

1.Dealey C. Cuidando de feridas: um guia para as enfermeiras. São Paulo: Atheneu; 2008.

2.Badke MR, Budó MLD, Silva FM, Ressel LB. Plantas medicinais: o saber sustentado na prática do cotidiano popular. Esc Anna Nery. 2011; 15:132-9.

3.Silva RS, Matos LSL, Araújo EC, Paixão GPN, Costa LEL, Pereira A. Práticas populares em saúde: autocuidado com feridas de usuários de plantas medicinais. Rev enferm UERJ. 2014; 22:389-95.

4.Piriz MA, Ceolin T, Mendieta MC, Mesquita MK, Lima CAB, Heck RM. O cuidado à saúde com o uso de plantas medicinais: uma perspectiva cultural. Ciênc cuid saúde. 2014; 13:309-17.

5.Sena J, Soares MCF, Cezar-Vaz MR, Sena A, Mucillo-Baisch AL. Visão docente sobre plantas medicinais como um saber e sua utilização como medicamento. Rev enferm UERJ. 2006; 14:196-201.

6.Geertz C. A interpretação das culturas. Rio de Janeiro: LTC; 2011.

7.Kleinman A. Patients and healers in the context of culture: an exploration of the bordeland between anthropology, medicine and psychiatry. California (USA): Regents; 1980. 8.Kleinman A. Concepts and a model for the comparison of medical systems as cultural systems. Soc Sci Med. 1978; $12(2 \mathrm{~B}): 85-95$.

9.Minayo MCS. O desafio do conhecimento: pesquisa qualitativa em saúde. 12a ed. São Paulo: HUCITEC; 2010. 10.Ministério da Saúde (Br). Conselho Nacional de Saúde. Resolução n ${ }^{\circ}$ 466/12: Normas regulamentadoras de pesquisa envolvendo seres humanos. Brasília (DF): Ministério da Saúde; 2012.

11.Ejaz S, Chekarova I, Cho JW, Lee SY, Ashraf S, Lim CW. Effect of aged garlic extract on wound healing: a new frontier in wound management. Drug Chem Toxicol. 2009; 32:191-203.

12.Zillmer JGV, Arrieira IC, Ceolin T, Heck RM, Barbieri RL . Utilização da babosa no cotidiano de usuários portadores de câncer. RBSP. 2010; 34:773-82.

13.Hashemi SA, Madani SA, Abediankenari S. The review on properties of Aloe Vera in healing of cutaneous wounds. Biomed Res Int. 2015; 2015:1-6.

14.Gentil M, Pereira JV, Sousa YT, Pietro R, Vansan LP, de 
Castro França S. In vitro evaluation of the antibacterial activity of Arctium lappa as a phytotherapeutic agent used in intracanal dressings. Phytother Res. 2006; 20(3):184-6. 15.Pereira JV, Bergamo DC, Pereira JO, França SC, Pietro RC, Silva-Sousa YT. Antimicrobial activity of Arctium lappa constituents against microorganisms commonly found in endodontic infections. Braz Dent J. 2005; 16:192-6.

16.Okuma CH, Andrade TA, Caetano GF, Finci LI, Maciel NR, Topan JF, et al. Development of lamellar gel phase emulsion containing marigold oil (Calendula officinalis) as a potential modern wound dressing. Eur J Pharm Sci. 2015; 71:62-72.

17.Nazar AK, Khalaf FH, Al-Zuhairi AH, Al-Ezzy AI. Evaluation of the Performance of Melia Azedarach for skin wound healing in donkeys: clinical and histopathological study. AJPCT. 2015; 3:1-9.

18.Stepán J, Ehrlichova J, Hladikova M. Efficacy and safety of symphytum herb extract cream in the treatment of pressure ulcers. Z Gerontol Geriatr. 2014; 47:228-35 19. Wu XB, Luo XQ, Gu SY, Xu JH. The effects of Polygonum cuspidatum extract on wound healing in rats. J Ethnopharmacol. 2012; 141:934-7.

20.Hitesh HK, Gupta MK. Wound healing activity of Polygonum barbatum Linn. J Pharm Pharmaceut Sci. 2012; 1:1084-91.

21.Lorenzi H, Matos FJA. Plantas medicinais no Brasil: nativas e exóticas. São Paulo: Instituto Plantarum; 2008. 22.Baggio CH, Freitas CS, Otofuji GM, Cipriani TR, Souza LM, Sassaki GL, at al. Flavonoid-rich fraction of Maytenus ilicifolia Mart. ex. Reiss protects the gastric mucosa of rodents through inhibition of both $\mathrm{H}+, \mathrm{K}+$-ATPase activity and formation of nitric oxide. J Ethnopharmacol. 2007; 113:433-40.

23.Cipriani TR, Mellinger CG, de Souza LM, Baggio CH, Freitas CS, Marques MC, et al. A polysaccharide from a tea (infusion) of Maytenus ilicifolia leaves with anti-ulcer protective effects. J Nat Prod. 2006; 69:1018-21.

24.Ojewole JAO. Antinociceptive, anti-inflammatory and antidiabetic effects of Bryophyllum pinnatum (Crassulaceae) leaf aqueous extract. J ethnopharmacol. 2005; 99:13-9. 25.Schapoval EE, Vargas MR, Chaves CG, Bridi R, Zuanazzi JA, Henriques AT. Antiinflammatory and antinociceptive activities of extracts and isolated compounds from Stachytarpheta cayennensis. J Ethnopharmacol. 1998; 60:53-9.

26.Okoye TC, Akah PA, Okoli CO, Ezike AC, Mbaoji FN. Antimicrobial and antispasmodic activity of leaf extract and fractions of Stachytarpheta cayennensis. Asian Pac J Trop Med. 2010; 3:189-92.

27.Ahmad M, Ansari MN, Alam A, Khan TH. Oral dose of citrus peel extracts promotes wound repair in diabetic rats. Pak J Biol Sci. 2013; 16:1086-94.

28.Pirbalouti AG, Azizi S, Koohpayeh A, Hamedi B. Wound healing activity of Malva sylvestris and punica granatum in alloxan-induced diabetic rats. Acta Pol Pharm. 2010; 67:511-6.

29.Barata LES, Alencar AAJ, Tascone M, Tamashiro J. Plantas medicinais brasileiras: I. Achyrocline satureioides (Lam.), DC. (Macela). Rev Fitos. 2009; 4:120-5.

30.Simões CMO. Farmacognosia: da planta ao medicamento. $6^{\mathrm{a}}$ ed. Porto Alegre (RS): Editora UFRGS; 2007. 31.Gould AN, Penny CB, Patel CC, Candy GP. Enhanced cutaneous wound healing by Senecio serratuloides (Asteraceae/Compositae) in a pig model. S Afri J Bot. 2015; 100:63-8.

32.Roersch CM. Piper umbellatum L.: a comparative cross-cultural analysis of its medicinal uses and an ethnopharmacological evaluation. J Ethnopharmacol. 2010; 131:522-37.

33.Nwauzoma AB, Dawari SL. Studies on the phytochemical properties and proximate analysis of Piper umbellatum (linn) from Nigeria. AJRC. 2013; 1(7):165-77.

34.Marongiu B, Alessandra PSP, Casu R, Pierucci P. Chemical composition of the oil and supercritical CO2 extract of Schinus molle L. Flavour Fragr J Flora. 2004; 19:554-8. 35.Palmeiro NS, Almeida CE, Ghedini PC, Goulart LS, Baldisserotto B. Analgesic and anti-inflammatory properties of plantago australis hydroalcoholic extract. Acta farm bonaer. 2002; 21(2):89-92.

36. Westerhof W, Das PK, Middelkoop E, Verschoor J, Storey L, Regnier C. Mucopolysaccharides from psyllium involved in wound healing. Drugs exp clin res. 2001; $27(5-6): 165-75$.

37.Zubair M. Genetic and environmental effects on polyphenols in Plantago major. Balsgård: Swedish University of Agricultural Sciences; 2010 [cited in 2013 jan 12]. Available from: http://pub.epsilon.slu.se/5468/1/ zubair_m_101022.pdf.

38.Chiang LC, Chiang W, Chang MY, Lin CC. In vitro cytotoxic, antiviral and immunomodulatory effects of Plantago major and Plantago asiatica. Am J Chin Med. 2003; 31(225):225-34.

39. Agência Nacional de Vigilância Sanitária (Br). Resolução - RDC n ${ }^{\circ}$ 10, de 9 de março de 2010 [acesso em 12 out 2014]. Disponível em: http://www.brasilsus.com.br/ legislacoes/rdc/103202-10.

40.Morais GFC, Oliveira SHS, Soares MJGO. Avaliação de feridas pelos enfermeiros de instituições hospitalares da rede pública. Texto contexto - enferm. 2008; 17:98-105. 